\title{
Tricobezoar gastroduodenal en la edad escolar. Caso clínico
}

\author{
Gastroduodenal Trichobezoar in school age. Case report
}

\author{
Dra. Edna L. Castrillón Peña ${ }^{a}$, Dr. Mario F. Espinosa Moreno ${ }^{b}$, Dr. Juan C. Barrios Torres ${ }^{b} y$ \\ Dr. Edgar E. Forero Niño ${ }^{c}$
}

\begin{abstract}
RESUMEN
Los bezoares son acumulaciones de sustancias orgánicas en el tracto gastrointestinal. El tricobezoar hace referencia a la acumulación de cabello, por logeneral, en el estómago eincluso más allá de este (síndrome de Rapunzel). La incidencia de esta enfermedad es extremadamente rara; el $90 \%$ de los casos se presentan en las mujeres, y el $80 \%$ de estos ocurren en menores de 30 años y se encuentran relacionados con conductas de ansiedad y trastornos psiquiátricos. El abordaje integral en estos pacientes es fundamental, junto con el seguimiento interdisciplinario, para tratar las causas psicoemocionales subyacentes y realizar una adecuada psicoeducación con los pacientes y sus familiares, para disminuir el riesgo de posibles recidivas. Se presenta el caso clínico de una paciente en edad escolar, con antecedente de maltrato físico por parte de su padrastro y posteriores conductas ansiosas (tricotilomanía y tricofagia).

Palabras clave: ansiedad, maltrato infantil, bezoares, tricofagia, tricotilomanía.
\end{abstract}

\begin{abstract}
Bezoar is an accumulation of organic substances in the gastrointestinal tract; trichobezoar refers to the accumulation of hair usually in the stomach and even beyond it (Rapunzel syndrome). The incidence of this condition is extremely rare, $90 \%$ of cases occur in women, and $80 \%$ of these occur in those under 30 years of age and are related to anxiety behaviors and psychiatric disorders. The integral approach in these patients is fundamental, together with the interdisciplinary follow-up, treating the underlying psycho-emotional causes and adequate psychoeducation with the patients and their relatives, in order to reduce the risk of possible recurrences. We present the clinical case of a patient of school age, with a history of physical abuse by her stepfather and subsequent anxious behaviors (trichotillomania and trichophagia).

Key words: anxiety, child abuse, bezoars, trichophagia, trichotillomania.
\end{abstract}

http:/ / dx.doi.org/10.5546/ aap.2019.e284

a. Salas de Cirugía, Clínica Medilaser, Neiva, Colombia.

b. Clínica Emcosalud, Neiva, Colombia.

c. Hospital Universitario Hernando Moncaleano Perdomo y Clínica Medilaser S. A., Neiva, Colombia.

Correspondencia:

Dr. Juan C. Barrios Torres: juancabarto@gmail.com

Financiamiento: Ninguno.

Conflicto de intereses: Ninguno que declarar.

Recibido: 27-7-2018

Aceptado: 1-1-2019
Cómo citar: Castrillón Peña EL, Espinosa Moreno MF, Barrios Torres JC, Forero Niño EE. Tricobezoar gastroduodenal en la edad escolar. Caso clínico. Arch Argent Pediatr 2019;117(3):e284-e287.

\section{INTRODUCCIÓN}

Un bezoar es la acumulación de sustancias orgánicas dentro del tracto gastrointestinal. El tricobezoar es una acumulación de cabello, generalmente, en las porciones proximales del tracto gastrointestinal, y es una enfermedad rara.

El término bezoar deriva de la palabra bazahr, que era usada por los árabes para describir antídotos de venenos extraídos de los animales, y trico deriva de la palabra griega trich, que significa 'cabello'."

La formación del tricobezoar comienza con la acumulación de cabello en los pliegues gástricos, el cual es altamente resistente a la digestión y a la peristalsis, debido a su superficie lisa. ${ }^{2} \mathrm{La}$ ingestión crónica lleva a su agrupación junto con otras sustancias presentes, como moco y alimento, y forma un cuerpo extraño que, generalmente, se limita al estómago. En algunos casos, este puede extenderse hasta el intestino o el colon. ${ }^{3}$

Los materiales que componen los bezoares comprenden material vegetal (fitobezoar), que es el tipo más común de bezoares; restos de frutas (diospirobezoares), que es el subtipo de fitobezoar más común; cabello (tricobezoar) y medicamentos ingeridos (farmacobezoar). ${ }^{4}$ Las personas con riesgo de desarrollar esta patología son aquellas con alteraciones anatómicas o en la motilidad gastrointestinal, como pacientes diabéticos, con patologías neurológicas, alteraciones en la secreción de jugo gástrico o con cirugías gástricas previas. ${ }^{5}$ Las enfermedades psiquiátricas y el retardo mental se asocian con los bezoares, debido a la pica y a la ingesta compulsiva y excesiva de comida. Los tricobezoares se presentan más en las mujeres, entre las adolescentes y las adultas jóvenes. ${ }^{6}$

A continuación, se presenta el caso de una paciente en edad escolar con antecedente de maltrato físico infantil y tricofagia, quien ingresó al Servicio de Urgencias por dolor abdominal crónico y sensación de masa abdominal. 


\section{PRESENTACIÓN DEL CASO}

Paciente femenina de 6 años quien consultó al Servicio de Urgencias en compañía de su abuela paterna por un cuadro clínico de dolor abdominal de 6 meses de evolución intermitente de tipo cólico en el hipocondrio izquierdo y el mesogastrio asociado a halitosis, flatos fétidos, distensión abdominal y sensación de masa referida por la abuela. No se asoció a vómitos ni a fiebre.

La paciente presentaba tricotilomanía y tricofagia desde los 18 meses de edad, asociadas a actos de violencia y maltrato, ligados a una disfunción familiar grave por ser producto de una madre adolescente y recibir maltrato físico infantil por su padrastro. En la consulta de Urgencias, esta se encontraba bajo custodia provisional de su abuela. En la evaluación por Trabajo Social, se encontró una familia nuclear recompuesta, funcional, activa, con la paciente en ciclo vital, en el período de edad escolar.

$\mathrm{Al}$ momento del examen físico, se encontraron áreas de alopecia en el cuero cabelludo, en la región temporoparietal bilateral, pérdida de masa muscular y tejido celular subcutáneo. En la exploración, se halló el abdomen distendido, con dolor ante la palpación en el hipocondrio izquierdo, con masa móvil entre el epigastrio y el hipocondrio izquierdo. El resto fue normal. Al momento del examen mental realizado por Psiquiatría, la paciente se encontró con afecto triste y ansioso de fondo, en su pensamiento, con sentimientos de nostalgia y abandono por parte de su madre biológica, con juicio disminuido en la capacidad de comprensión. Describió que no le gustaba ver escenas de maltrato. No se observaron cambios motores.

Posteriormente, fue evaluada por el Servicio de Pediatría, quienes solicitaron una ecografía abdominal total (Figura 1), que sugirió un tricobezoar. Se requirió la evaluación por Cirugía Pediátrica, quienes ordenaron la toma de una endoscopía de las vías digestivas altas y confirmaron el diagnóstico de tricobezoar. Se intentó la extracción endoscópica, que no fue exitosa por no lograrse el avance hasta más

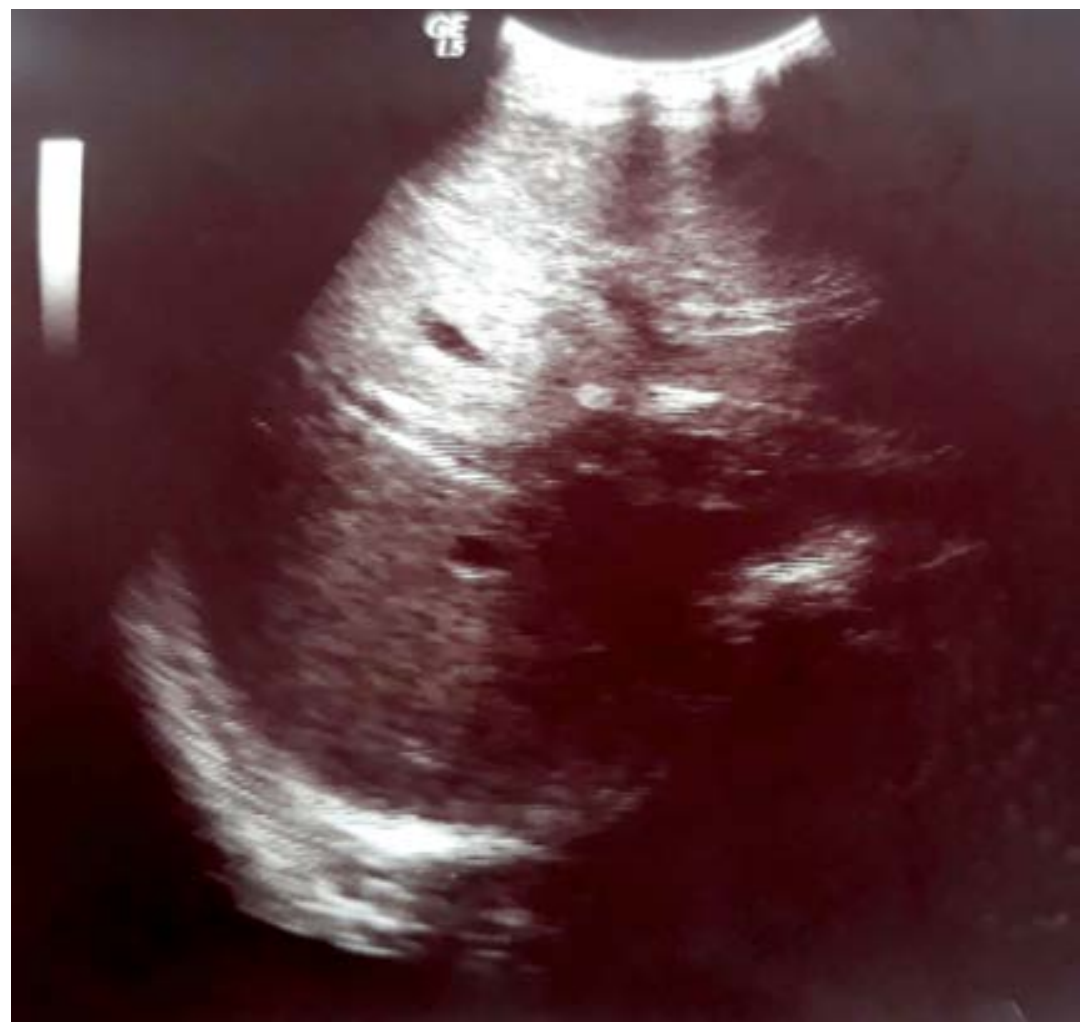


allá del esófago, debido al gran tamaño del tricobezoar, que obstruía alrededor del $80 \%$ de la luz del tracto gastrointestinal. Se realizaron análisis paraclínicos de control, como hemograma, transaminasas, bilirrubinas y uroanálisis, que fueron normales.

Una vez realizado el diagnóstico imagenológico concordante con la clínica, se decidió llevar a la extracción quirúrgica mediante gastrotomía abierta y se encontró un tricobezoar gigante de $6 \times 6 \times 20$ centímetros con ocupación completa del cuerpo gástrico y parcial del duodeno (Figura 2).

Fue evaluada por el equipo interdisciplinario de Psiquiatría, Psicología, Trabajo Social y Nutrición, quienes realizaron psicoeducación. Psiquiatría inició el manejo farmacológico para el control de la ansiedad con fluoxetina a razón de 10 miligramos al día. En el posoperatorio, no presentó ninguna complicación, por lo que se dio el egreso con manejo farmacológico para su ansiedad y controles ambulatorios con las especialidades tratantes.

Actualmente, la paciente continúa en seguimiento interdisciplinario por el equipo de Pediatría y Psiquiatría para el manejo de la ansiedad asociada a la tricotilomanía y tricofagia, con una adecuada ganancia pondoestatural y disminución de los episodios ansiosos.

\section{DISCUSIÓN}

Los bezoares gástricos son entidades infrecuentes en las edades pediátricas. La mayoría de los casos corresponden a tricobezoares, que son hallazgos de cabello encontrados en el estómago secundarios a la tricotilomanía y a la tricofagia. ${ }^{7} \mathrm{La}$ incidencia de esta enfermedad es rara y solo se han reportados algunos casos en el mundo; ${ }^{8}$ el $90 \%$ se presentan en mujeres, y el $80 \%$ de estos ocurren en menores de 30 años, ${ }^{9}$ a diferencia de nuestro caso, en el que llama la atención que ocurre en una paciente de sexo femenino en edad escolar. En la literatura, hay muy pocos casos reportados en este grupo etario. En nuestro medio, no existen estudios que evalúen la frecuencia de esta enfermedad. En general, los bezoares se pueden encontrar en el estómago en menos del 0,5\% de los individuos sometidos a endoscopías de las vías digestivas altas. Sin embargo, la prevalencia de bezoares, probablemente, varía entre grupos étnicos y ubicaciones geográficas. ${ }^{10}$

El espectro clínico de estos pacientes es variable; la mayoría de ellos, en el inicio, 
pueden permanecer asintomáticos por largos períodos. Los síntomas, típicamente, comprenden zonas de alopecia, dolor abdominal, náuseas, vómitos, pérdida de peso, anorexia, saciedad temprana y síntomas relacionados con la anemia, como mareo, palpitaciones y cansancio. ${ }^{1}$ Esta presentación es similar a la encontrada en nuestro caso, dado que la paciente presentaba, al momento de la inspección, áreas de alopecia que indicaron la tricotilomanía y la posible tricofagia, asociadas al dolor abdominal crónico, por el que había consultado previamente, pero sin un diagnóstico claro. Al momento de la realización del diagnóstico, se presentaban náuseas y pérdida de peso, aunque sin alteraciones relacionadas con síntomas anémicos.

Las masas palpables en el abdomen también se encuentran dentro del espectro clínico. ${ }^{11}$ Este hallazgo fue fundamental en nuestro caso para realizar un correcto abordaje clínico y el posterior diagnóstico.

Para el diagnóstico de esta entidad, se debe iniciar con un abordaje adecuado del paciente y una historia clínica completa. Dentro de los estudios imagenológicos, está la ecografía de abdomen, en la cual se puede observar un área ecogénica con sombra acústica posterior. También en la tomografía computarizada de abdomen, se pueden detectar bezoares gástricos y del intestino delgado, al visualizar una masa en el tracto gastrointestinal con burbujas de aire retenidas y con apariencia moteada. ${ }^{10}$ Sin embargo, el estándar de oro lo constituye la endoscopía de las vías digestivas altas, que desempeña un papel fundamental en su diagnóstico y tratamiento cuando es posible. ${ }^{12}$

El abordaje integral en estos pacientes es fundamental. Inicialmente, se intenta la extracción endoscópica, siempre y cuando sea factible. En aquellos casos en los que no lo es, el abordaje quirúrgico se convierte en el tratamiento de elección. En nuestra paciente, se intentó la extracción endoscópica sin tener éxito por la falta de avance esofágico. Se recurrió a la gastrotomía, ${ }^{13}$ debido al tamaño del tricobezoar, por las posibles complicaciones al intentar un abordabje laparoscópico, como la perforación de víscera hueca, lesión de órgano sólido, lesión vascular mayor y neumoperitoneo residual doloroso. Además del manejo quirúrgico, el abordaje por el equipo de Psiquiatría es fundamental para prevenir la recurrencia de estos casos, la cual alcanza cifras de hasta el $20 \%$ si no se controlan los factores de ansiedad, como la tricofagia.
Por ello, es importante identificar las causas asociadas al trastorno, que, en nuestro caso, fueron el maltrato físico previamente recibido por la paciente y las escenas asociadas a este, que causaban ansiedad. ${ }^{14}$

Finalmente, el seguimiento interdisciplinario se convierte en una importante herramienta para explorar y tratar las causas psicoemocionales subyacentes, así como la psicoeducación hacia los pacientes y sus familiares sobre los riesgos que conlleva la recidiva del cuadro clínico. En este caso, al tratarse de una paciente que se encuentra en un ciclo vital temprano, el fortalecimiento y la adecuada estructuración de una personalidad sólida son preponderantes para evitar posteriores recurrencias.

\section{REFERENCIAS}

1. Akbar S, Akbar L, Akbar N, Nawras A. TrichobezoarInduced Heartburn in a Teenage Girl: A Case Report. J Investig Med High Impact Case Rep. 2018; 6:2324709618776345.

2. Gorter R, Kneepkens C, Mattens E, Aronson D, et al. Management of trichobezoar: Case report and literature review. Pediatr Surg Int. 2010; 26(5):457-63.

3. Naik S, Gupta V, Naik S, Rangole A, et al. Rapunzel syndrome reviewed and redefined. Dig Surg. 2007; 24(3):157-61.

4. Couceiro A, Viveiro C, Capelão G, Nobre J, et al. Trichobezoar - a rare cause of abdominal mass and gastric outlet obstruction. GE Port J Gastroenterol. 2015; 23(1):50-3.

5. Phillips MR, Zaheer S, Drugas GT. Gastric trichobezoar: case report and literature review. Mayo Clin Proc. 1998; 73(7): 653-6.

6. Calabuig R, Navarro S, Carrió I, Artigas V, et al. Gastric emptying and bezoars. Am J Surg. 1989; 157(3):287-90.

7. Marique L, Wirtz M, Henkens A, Delchambre E, et al. Gastric Perforation due to Giant Trichobezoar in a 13-YearOld Child. J Gastrointest Surg. 2017; 21(6):1093-4.

8. Nettikadan A, RaviMJ, Shivaprasad M. Recurrent Rapunzel syndrome - A rare tale of a hairy tail. Int J Surg Case Rep. 2018; 45:83-6.

9. Bargas-Ochoa M, Xacur-Hernández M, Espadas-Torres M, Quintana Gamboa A, et al. Síndrome de Rapunzel con doble tricobezoar simultáneo en una adolescente: Reporte de caso. Rev Chil Pediatr. 2018; 89(1):98-102.

10. Iwamuro M, Okada H, Matsueda K, Inaba T, et al. Review of the diagnosis and management of gastrointestinal bezoars. World J Gastrointest Endosc. 2015; 7(4):336-45.

11. D’Amora M, Cremone G, Rossi E, Zeccolini M. A nonocclusive bezoar of caecum in a 7-year-old child: ultrasound detection and multimodality imaging management. J Ultrasound. 2015; 19(3):223-6.

12. Zhao J, Zhao W, Wang Y. Endoscopic retrieval of gastric trichophytobezoar: case report of a 12-year-old girl with trichophagia. Medicine (Baltimore). 2017; 96(3):e5969.

13. Coronel C, Martínez Arguello D, Cáceres R, Vera A. Síndrome de retención gástrica por tricobezoar. Rev Cir Parag. 2012; 36(2):30-2.

14. Frey AS, McKee M, King RA, Martin A. Hair apparent: Rapunzel syndrome. Am J Psychiatry. 2005; 162(2):242-8. 